\title{
Violência sexual na infância associa-se a qualidade de vida inferior em universitários
}

\author{
The sexual violence occurred in childhood is associated \\ with undergraduate student's life quality \\ Karla Julianne Negreiros de Mato\$ ${ }^{*}$, Francisco José Maia Pinto', Ana Carina Stelko-Pereira'
}

\section{RESUMO}

Objetivo: Analisar a existência de possível associação entre a exposição ao abuso sexual na infância e qualidade de vida inferior em universitários. Métodos: Participaram da pesquisa 935 estudantes universitários que responderam ao questionário de prevalência de abuso sexual infantil e ao questionário abreviado de avaliação de qualidade de vida (WHOQOL-bref), bem como a questões sociodemográficas e sobre histórico de doenças clínicas. Utilizou-se o teste de associação do qui-quadrado e análise de regressão logística, tendo-se como desfecho a variável binária qualidade de vida (inferior; superior). No modelo final de regressão, aplicou-se o teste de sensibilidade para identificar o acerto entre os casos observados com qualidade de vida superior e o teste de especificidade aplicado aos casos observados com qualidade de vida inferior, bem como avaliou-se a eficiência global do modelo para um cutoff de 0,5. Resultados: A maioria dos participantes era: mulher (55,6\%, 520), parda (52,3\%, 489), solteira $(58,4 \%, 546)$. A idade média foi de 21 anos $(S D= \pm 5)$ e a renda familiar média, de $R \$$ $3.875,00$ (SD $= \pm 4.690,00)$. A partir da regressão logística, observou-se que aumentaram a associação com qualidade de vida inferior: necessidade de acompanhamento psicológico, estar solteiro, sofrer violência sexual na infância, não ser de raça branca, praticar atividade física três vezes ou mais na semana, nascer no interior e praticar religião. Conclusão: $\bigcirc$ estudo evidenciou que o abuso sexual infantil, mesmo transcorrido há muitos anos, associa-se negativamente à qualidade de vida de adultos.

\section{Palavras-chave \\ Qualidade de vida, estudantes, abuso sexual infantil.}




\section{Keywords}

Quality of life, students, child sexual abuse. superior life quality and the specificity test applied to the cases observed with lower life quality. The overall efficiency percentage of the model was applied to a cutoff of 0.5. Results: The majority of participants were: female $(55.6 \%, 520)$, brown $(52.3 \%, 489)$, single $(58.4 \%$, 546). They were at the average age of 21 years old $(S D= \pm 5)$ and average family income of $R \$$ $3,875.00$ ( $S D= \pm 4,690.00$ ). From logistic regression, it was observed association with inferior life quality: need for psychological counseling, being single, suffering sexual violence in childhood, not being Caucasian, practicing physical activity three times or more a week, being born in rural area and practicing religion. Conclusion: The study showed that sexual abuse, even after many years, still has an association with inferior life quality of university students.

\section{INTRODUÇÃO}

O abuso sexual infantil (ASI) é apontado, pela Organização Mundial da Saúde (OMS), como uma das mais proeminentes questões de saúde pública, definindo-se por atos que envolvem coagir ou motivar crianças a participarem de práticas sexuais, tais como: obrigar a assistir a sexo ou pornografia, a masturbar-se em público, a posar eroticamente para fotos e filmes, a realizar toques e carícias sexuais e a praticar sexo genital, anal e oral. Portanto, o ASI engloba tanto situações sem contato físico como com contato físico, sem penetração e com penetração. 0 ofensor é um indivíduo que está em estágio de desenvolvimento superior à vítima e/ou em uma relação de responsabilidade, confiança ou poder em relação a ela. O ASI frequentemente é crônico, por ofensores que envolvem a criança em um processo gradual de sexualização e que costumam ser familiares das vítimas ou responsáveis por elas'. Para além do ASI, é possível também durante a infância e a adolescência sofrer violência sexual por pares², como por colegas de escola.

A violência sexual na infância e adolescência é um fenômeno frequente no Brasil e em outros países. Uma metanálise de 244 artigos sobre maus-tratos infantis apresentou a proporção de 127 pessoas vítimas de ASI a cada 1.000 pessoas; para meninos essa taxa é de 76/1.000 e para meninas, de 180/1.000. Nesse mesmo estudo, apresentou-se que na América do Sul cerca de 13\% de meninos e meninas são abusados sexualmente ${ }^{3}$. Um censo com escolares do nono ano do ensino fundamental realizado pelo Instituto Brasileiro de Geografia e Estatística em parceria com o Ministério da Educação e da Saúde indicou que 4\% responderam afirmativamente à questão "Alguma vez na vida você foi forçado a ter relação sexual?", e 32,6\% por familiares, dos quais 11,9\% por pai/mãe/padrasto, $26 \%$ por namorado/ex-namorado e 21,8\% por amigos². Outros estudos brasileiros também vêm demonstrando que as vítimas são predominantemente do gênero feminino e que grande parte das violências sexuais ocorre em ambiente familiar ${ }^{4,5}$.

É conhecido o fato de que a ASI acarreta efeitos negativos no desenvolvimento das crianças e dos adolescentes ${ }^{6}$ e que se reflete na vida adulta. Em revisão de literatura ${ }^{2}$, destaca-se que as crianças que sofreram maus-tratos na infância, entre eles abuso sexual, apresentaram sequelas que costumaram durar até a idade adulta, incluindo mudanças de longo prazo na estrutura cerebral, problemas de saúde mental e física, comportamentos de risco, problemas com o funcionamento social e redução da expectativa de vida. Ademais, é mais provável que as vítimas de maus-tratos na infância apresentem mais gastos com problemas de saúde e se aposentem precocemente ${ }^{7}$.

Existe a hipótese de que acontecimentos traumáticos sensibilizam o cérebro - especialmente durante o período da infância, em que há constantes transformações na organização cerebral -, desorganizando-o quimicamente e tornando seu funcionamento atípico e disfuncional. Quando crianças são expostas a eventos muito traumáticos (por exemplo, abusos físicos e sexuais intensos), os caminhos neurológicos que são responsáveis pelas respostas de "luta ou fuga" são superativados por um prolongado período de tempo ${ }^{8}$.

Embora haja estudos que apontem sequelas neurológicas e problemas mentais, como transtorno depressivo, de estresse pós-traumático e tentativas de suicídio ${ }^{6}$, às vítimas de abuso sexual na infância, poucos estudos evidenciaram o impacto na qualidade de vida (QV) de crianças que sofreram maus-tratos, principalmente após terem sido transcorridos vários anos ${ }^{9,10}$. Entende-se por QV a "percepção do indivíduo de sua posição na vida, no contexto da cultura e sistema de valores nos quais ele vive e em relação aos seus objetivos, expectativas, padrões e preocupações (p. 1405)", segundo a OMS ${ }^{11}$. Assim, este estudo se propôs a analisar a existência de possível associação entre a exposição à violência sexual na infância e uma qualidade de vida inferior (QVI) no período adulto, em adultos universitários, levando em consideração a influência conjunta de outras variáveis, como sexo e etnia. Hipotetizava-se que a violência sexual na infância se associaria a uma QVI na vida adulta.

\section{MÉTODOS}

\section{Delineamento e população do estudo}

Este estudo é do tipo transversal, com abordagem descritiva e analítica, teve como participantes 935 estudantes da Universidade Estadual do Ceará, do campus de Fortaleza, que é organizado em seis centros: Ciências da Saúde, da Educação, de Humanidades, de Estudos Sociais Aplicados, de Ciências 
e Tecnologia e Faculdade de Veterinária. Esses alunos representavam proporcionalmente cerca de $10 \%$ dos alunos de cada centro e do total de alunos da universidade, tendo sido convidados a participar pelo pesquisador, em suas salas de aula, em horário letivo. Assim, a amostra de participantes foi recrutada por conveniência. Houve 38 alunos que foram convidados, mas não aceitaram participar da pesquisa.

\section{Instrumentos}

Foram utilizados dois questionários de autorrelato, os quais foram aplicados coletivamente nas salas de aula dos participantes. Um deles é o Questionário de Prevalência de Violência Sexual na Infância, o qual foi elaborado por 12 pesquisadores e profissionais da área da psicologia forense, de diferentes regiões brasileiras, com titulação mínima de mestrado, todos vinculados a programas de pós-graduação, e compondo o grupo "Tecnologia Social e Inovação: Intervenções Psicológicas e Práticas Forenses contra Violência" da Associação de Pesquisa em Psicologia' ${ }^{2}$. O grupo reuniu-se ao longo de dois dias a fim de compor os itens do instrumento, que se compôs de 32 questões, divididas em três partes, das quais, para este estudo, apenas a primeira e a segunda foram utilizadas. A primeira parte contém sete perguntas sobre questões para identificar características sociodemográficas dos respondentes, como: "Com quem você mora?".

A segunda parte do questionário apresentava 13 questões sobre se o respondente vivenciou situações de abuso, seja sem contato físico, com contato físico, sem penetração e com penetração, até a idade de 14 anos, investigando a frequência dessas situações, com as opções de resposta: nunca, algumas vezes e muitas vezes. Cabe ressaltar que o(s) ofensor(es) podiam ou não estar em estágio de desenvolvimento superior ao da vítima e/ou em uma relação de responsabilidade. As perguntas dessa parte englobaram realizar comentários com conteúdo sexual inapropriado direcionados à criança, fazer gestos para a criança simulando masturbação ou sexo, obrigar a ver fotos eróticas ou fazer/enviar fotos eróticas, mostrar filmes com cenas de sexo em vídeo ou pela internet, requisitar que a criança fique despida por motivos sexuais, masturbar a criança ou solicitar masturbação, acariciar ou tocar genitais, introduzir objetos na vagina ou ânus, realizar ou receber sexo oral e anal, praticar sexo genital e prostituição. Com o banco de dados deste estudo, notou-se que o instrumento apresentava um índice de alfa de Cronbach de 0,89 quanto às questões de frequência, apresentando alta consistência interna.

Outro instrumento empregado foi o WHOQOL-bref, o qual é um instrumento de avaliação de QV que investiga a capacidade física (sete questões), o bem-estar psicológico (seis questões), as relações sociais (três questões) e o meio ambiente onde o indivíduo está inserido (oito questões). Esse instrumento apresenta evidências de validade e precisão em estudos realizados no Brasil|13-16. Ademais, empregaram-se questões elaboradas para o estudo a fim de caracterizar os participantes sociodemograficamente e quanto a doenças clínicas.

\section{Aspectos éticos}

Este estudo foi aprovado pelo Comitê de Ética em Pesquisa (CAEE 31448214.9.0000.5534), e todos os participantes assinaram o Termo de Consentimento Livre e Esclarecido. Ademais, após a coleta de dados, forneceu-se um folder ${ }^{17}$ para que os participantes se informassem sobre o fenômeno da violência sexual infantil e a Lei Joana Maranhão (Lei no 12.650/2012), a qual apresenta os direitos dos indivíduos adultos que foram abusados sexualmente quando crianças.

\section{Análise estatística}

Os dados foram digitados sem identificação dos participantes pela equipe de recursos humanos, tendo-se utilizado o SPSS versão 18.0 (Statistical Package for Social Science) para as análises. Foram realizadas análises descritivas quanto às características sociodemográficas e, em seguida, foram calculados os escores totais de QV de cada participante e o escore médio do grupo de participantes. Os indivíduos que tiveram escore total igual ou acima do valor correspondente à média mais um desvio-padrão (74,1 pontos) foram classificados como com qualidade de vida superior (QVS), enquanto os indivíduos que apresentaram escore igual ou abaixo da média menos um desvio-padrão (49,9 pontos) foram categorizados como com QVI. Os indivíduos que obtiveram escores de QV entre 50 e 74, isto é, que não foram classificados como tendo QV nem inferior e tampouco superior, ou seja, que tiveram um escore próximo à média da população total de 935 alunos, foram excluídos das análises subsequentes (639 estudantes).

As análises posteriores envolveram as variáveis: idade (menos de 20 anos e mais de 21 anos), sexo (masculino, feminino), etnia (branca, não branca), conjugalidade (com companheiro, sem companheiro), prática religiosa (sim, não), local de nascimento (capital, interior), se exerce atividade remunerada (sim, não), se sofreu acidente grave (sim, não), se pratica atividade física no mínimo três vezes na semana (sim, não), se possui doença crônica (sim, não), se recebe atendimento psiquiátrico (sim, não), se recebe atendimento psicológico (sim, não) e se fez uso de medicação psiquiátrica (sim, não). Ademais, incluiu-se a variável se foi vítima de violência sexual na infância (sim ou não), tendo-se considerado como vítima os respondentes que não marcaram no Questionário de Prevalência de Abuso Sexual, em sua segunda parte, a resposta "nunca" para qualquer uma das 13 questões sobre envolvimento em abuso sexual. Essas análises subsequentes foram o teste de associação do qui-quadrado de Pearson, cruzando-se a variável QV (inferior ou superior) com cada uma das outras variáveis já descritas e, na 
impossibilidade dele, o da razão de máxima verossimilhança, em nível de significância de 5\%.

Seguidamente a essas análises, fez-se análise de regressão logística para quantificar a associação entre QVI e as outras variáveis do estudo, conjuntamente, por meio da razão de chances. Para a análise de regressão, exclui-se os participantes que deixaram de preencher qualquer uma das questões relativas a idade, sexo, etnia, conjugalidade, prática religiosa, local de nascimento, se exerce atividade remunerada, se sofreu acidente grave, se pratica atividade física, se possui doença crônica, se recebe atendimento psiquiátrico, se recebe atendimento psicológico, se fez uso de medicação psiquiátrica e se foi vítima de violência sexual na infância. Assim, para essa análise, a quantidade de participantes foi de 245, dos quais 128 tiveram QV superior e 117 inferior.

Por meio do teste de Hosmer-Lemeshow, rejeitou-se a hipótese nula de que as frequências esperadas e observadas seriam iguais. Consideram-se como entrada no modelo as variáveis que em análise por qui-quadrado apresentaram $p<$ 0,20 e, para permanecerem no modelo, apenas aquelas que apresentaram $p<0,05$. O método de seleção das variáveis do modelo de regressão foi o de seleção manual, a fim de levar em conta o que a literatura já apontava como variáveis de maior influência na QV. No modelo final de regressão, aplicou-se o teste de sensibilidade para identificar o acerto, entre os casos observados com QVS, e o teste de especificidade aplicado aos casos observados com QVI. Aplicou-se o percentual de eficiência global do modelo para um cutoff de 0,5.

\section{RESULTADOS}

O perfil dos 935 participantes foi: a maioria dos participantes era do sexo feminino $(55,6 \%, 520)$, parda $(52,3 \%, 489)$, solteira $(58,4 \%, 546)$, morando com a mãe $(75,7 \%, 708)$. Houve a predominância dos pais e mães com escolaridade máxima até o ensino fundamental - 37,6\% (326) e 36,9\% (306), respectivamente. A renda média familiar foi de $\mathrm{R} \$ 3.875,00$ (SD $\pm 4.690,00)$. A média de idade foi de 21 anos (SD \pm 5 ), sendo o mais jovem de 16 anos e o mais velho de 63 anos.

A média de $\mathrm{QV}$ dos participantes foi de $62,4(\mathrm{DP}= \pm 12,15)$, sendo 16,2\% (152) classificados como com QVI, 15,5\% (144) com QVS e 68,3\% (639) com QV mediana, sendo os com QV mediana descartados das análises subsequentes. Dos que apresentavam QV inferior, a maioria $(63,1 \%, 101)$ foi vítima de abuso sexual na infância, seja este com ou sem penetração, enquanto, dos que apresentavam QVS, 36,9\% (59) foram vítimas dessa mesma violência.

A partir do qui-quadrado (Tabela 1), observou-se que houve diferença significativa entre os grupos com QV inferior e superior quanto a: etnia, conjugalidade, realizar atividade física três vezes ou mais por semana, ser portador de doença crônica, ter tido atendimento psiquiátrico e/ou psicológico, usar medicação psiquiátrica e ter sofrido violência sexual na infância.

Foram realizados 10 blocos na análise de regressão logística binária até se estabelecer o modelo final, o qual considerou conjuntamente as diversas variáveis do estudo a fim de predizer QVI. Por meio do Teste Omnibus, verificou-se a hipótese quanto a se os coeficientes do modelo de regressão seriam nulos, tendo-se obtido o valor do qui-quadrado de 71.321 (g.l. = 7) com significância menor que 0,001. Por meio do teste de Hosmer-Lemeshow, rejeitou-se a hipótese nula de que as frequências esperadas e observadas seriam iguais - qui-quadrado 3.009 (g.l. = 7) com significância menor de 0,884.

O modelo final de regressão elaborado permitiu classificar dos 128 casos observados como QV superior, 96 corretamente, representando 75,0\% de acerto (sensibilidade). Dos 117 casos observados como QVI, 82 foram classificados corretamente, representando $70,1 \%$ de acerto (especificidade). O percentual de eficiência global do modelo foi de 72,7\% para um cutoff de 0,5 . De acordo com a estatística Z de Wald e o nível de significância adotado de 0,05 , constatou-se que alguns coeficientes foram estatisticamente diferentes de zero (Tabela 2).

Após análise de regressão logística, as variáveis que se mantiveram associadas a QVI em ordem decrescente de importância, conforme a Tabela 2, foram: a) ter necessitado de atendimento psicológico (3,78 mais associado com QVI do que quem não necessitou); b) estar solteiro ou sem companheiro (2,43 mais associado com QVI do que com companheiro); c) ter sido vítima de abuso sexual na infância (1,55 mais relacionado com QVI do que quem não foi vítima); d) não ser branco (1,53 mais relacionado com QVI do que quem é branco); e) praticar atividade física três vezes ou mais na semana (0,67 mais associado com QVI do que quem não pratica); $f$ ) morar no interior $(0,57$ mais associado com QVI do que quem nasceu na capital); g) quem se diz com religião $(0,49$ mais relacionado com $\mathrm{QVI}$ do que quem afirma não ter religião). As variáveis que não chegaram ao modelo final, mas que haviam sido significativas em análise de qui-quadrado foram: ter doença crônica, ter necessidade de acompanhamento psiquiátrico e utilizar de medicações psiquiátricas, de modo que, quando consideradas conjuntamente com as outras variáveis investigadas neste estudo, não contribuíram para predizer QVI em universitários.

\section{DISCUSSÃO}

Este estudo teve como objetivo analisar a existência de possível associação entre a exposição à violência sexual na infância e uma QVI na vida adulta, tendo como participantes universitários, e considerando conjuntamente as variáveis: idade, sexo, etnia, conjugalidade, prática religiosa, local de nascimento, exercício de atividade remunerada, sofrer acidente grave, praticar atividade física, possuir doença crônica, 
Tabela 1. Análise bruta entre qualidade de vida e as características sociais, demográficas, econômicas e histórico de doenças clínica de universitários (Universidade Estadual do Ceará - UECE, 2014)

\begin{tabular}{|c|c|c|c|c|c|}
\hline & \multicolumn{2}{|c|}{ Qualidade de vida } & \multirow{2}{*}{$x^{2}$} & \multirow{2}{*}{$p$} & \multirow{2}{*}{ OR (IC95\%) } \\
\hline & Inferior \%(N) & Superior \% (N) & & & \\
\hline \multicolumn{6}{|l|}{ Etnia } \\
\hline Não brancos & 59 (109) & $41(76)$ & 11,87 & 0,001 & $2,32(1,43-3,76)$ \\
\hline Brancos & $38(42)$ & $62(68)$ & & & \\
\hline \multicolumn{6}{|l|}{ Gênero } \\
\hline Masculino & $47(66)$ & $53(76)$ & 2,59 & 0,130 & $0,68(0,43-1,08)$ \\
\hline Feminino & $60(86)$ & $40(68)$ & & & \\
\hline \multicolumn{6}{|l|}{ Conjugalidade } \\
\hline Sem companheiro & $60(111)$ & $40(72)$ & 16,61 & $<0,001$ & $2,70(1,66-4,39)$ \\
\hline Com companheiro & $36(41)$ & $64(72)$ & & & \\
\hline \multicolumn{6}{|l|}{ Idade } \\
\hline Até ou com 20 anos & $55(65)$ & $45(53)$ & 0,79 & 0,390 & $1,24(0,77-2,00)$ \\
\hline Acima de 21 anos & $49(79)$ & $51(80)$ & & & \\
\hline \multicolumn{6}{|l|}{ Religiosidade } \\
\hline Não ter religião & $43(67)$ & $57(90)$ & 5,090 & 0,024 & $0,55(0,33-0,92)$ \\
\hline Ter religião & $57(55)$ & $43(41)$ & & & \\
\hline \multicolumn{6}{|l|}{ Local nascimento } \\
\hline Capital & $61(33)$ & $39(21)$ & 2,51 & 0,120 & $1,62(0,88-2,96)$ \\
\hline Interior & 49 (119) & $51(123)$ & & & \\
\hline \multicolumn{6}{|l|}{ Atividade remunerada } \\
\hline Trabalha & $49(80)$ & $51(84)$ & 0,75 & 0,412 & $0,81(0,51-1,29)$ \\
\hline Não trabalha & $54(70)$ & $46(60)$ & & & \\
\hline \multicolumn{6}{|l|}{ Vítima de acidente* } \\
\hline Sofreu acidente & $45(8)$ & $55(10)$ & 0,31 & 0,630 & $0,76(0,29-1,98)$ \\
\hline Não sofreu acidente & $51(140)$ & 49 (133) & & & \\
\hline \multicolumn{6}{|c|}{ Atividade física três ou mais vezes na semana } \\
\hline Sem atividade física & $41(53)$ & $59(76)$ & 10,75 & 0,001 & $0,48(0,28-0,73)$ \\
\hline Com atividade física & $60(99)$ & $40(65)$ & & & \\
\hline \multicolumn{6}{|c|}{ Portador de doença crônica* } \\
\hline Com doença crônica & $70(25)$ & $30(11)$ & 5,37 & 0,022 & $2,38(1,12-5,07)$ \\
\hline Sem doença crônica & $49(127)$ & $51(133)$ & & & \\
\hline \multicolumn{6}{|c|}{ Atendimento psiquiátrico* } \\
\hline Sem necessidade & $48(132)$ & $52(141)$ & 11,77 & 0,001 & $0,14(0,43-0,51)$ \\
\hline Com necessidade & $14(3)$ & $86(19)$ & & & \\
\hline \multicolumn{6}{|c|}{ Atendimento psicológico* } \\
\hline Sem necessidade & $46(111)$ & $54(132)$ & 16,73 & $<0,001$ & $0,25(0,12-0,50)$ \\
\hline Com necessidade & $77(40)$ & $23(12)$ & & & \\
\hline \multicolumn{6}{|c|}{ Uso de medicação psiquiátrica* } \\
\hline Não utiliza & $48(132)$ & $52(141)$ & 11,77 & 0,001 & $0,15(0,43-0,51)$ \\
\hline Utiliza & $86(19)$ & $14(3)$ & & & \\
\hline \multicolumn{6}{|l|}{ Portador de deficiência* } \\
\hline Com deficiência & $75(3)$ & $25(1)$ & 0,94 & 0,623 & $2,93(0,30-28,58)$ \\
\hline Sem deficiência & $51(146)$ & 49 (134) & & & \\
\hline \multicolumn{6}{|l|}{ Abuso sexual infantil } \\
\hline Vítima & 63 (101) & $37(59)$ & 20,72 & $<0,001$ & $2,99(1,85-4,83)$ \\
\hline Não vítima & $36(48)$ & $63(84)$ & & & \\
\hline
\end{tabular}

Significância estatística pelo qui-quadrado $(p<0,05)$. * Fisher's Exact Test.

receber atendimento psiquiátrico e psicológico e usar medicação psiquiátrica.

Os participantes deste estudo apresentaram semelhanças com a população brasileira, a qual se refere a uma maioria de mulheres (51\%), e o percentual neste estudo foi de $55 \%$. Quanto à idade dos participantes, verificou-se que tinham em média 20 anos; esses se incluem no grupo de cerca de $49,68 \%$ dos brasileiros que possuem até 24 anos $^{11}$. Quanto à etnia, o IBGE ${ }^{18}$ apresenta que no Brasil $47,7 \%$ se dizem brancos, 43,1\% pardos, enquanto, entre os participantes deste estudo, $40 \%$ se declararam brancos e $51 \%$ pardos. Quanto à renda familiar média dos participantes deste estudo, essa envolveu cerca de cinco salários mínimos, de modo que os participantes se incluiriam nos $70 \%$ dos brasileiros que possuem renda familiar superior a três salários mínimos.

A prevalência de violência sexual infantil encontrada foi bastante superior à de estudos internacionais e nacionais, possivelmente porque nesta investigação se consideraram não somente situações de abuso sexual com contato físico e/ou com penetração, mas também situações sem contato, como expor a pornografia ou sexo, obrigar a masturbar-se diante do ofensor, entre outras ${ }^{1,2}$. Destaca-se também que o(s) ofensor(es) po- 
Tabela 2. Modelo final ajustado pela regressão logística entre a qualidade de vida e as variáveis explicativas ( $N=245)($ Universidade Estadual do Ceará - UECE, 2014)

\begin{tabular}{|c|c|c|c|c|c|c|}
\hline & B & $B(S E)$ & Wald & df & $p$ & $\operatorname{Exp}(B)$ \\
\hline $\begin{array}{l}\text { Atendimento psicológico } \\
\text { Com necessidade } \\
\text { Sem necessidade }\end{array}$ & 1,564 & 0,454 & 11,857 & 1 & 0,001 & $\begin{array}{c}4,78 \\
(1,962-11,64)\end{array}$ \\
\hline $\begin{array}{l}\text { Conjugalidade } \\
\text { Sem companheiro } \\
\text { Com companheiro }\end{array}$ & 1,235 & 0,318 & 15,062 & 1 & $<0,001$ & $\begin{array}{c}3,439 \\
(1,843-6,418)\end{array}$ \\
\hline $\begin{array}{l}\text { Abuso sexual infantil } \\
\text { Vítima } \\
\text { Não vítima }\end{array}$ & 0,939 & 0,309 & 9,229 & 1 & 0,002 & $\begin{array}{c}2,559 \\
(1,396-4,690)\end{array}$ \\
\hline $\begin{array}{l}\text { Etnia } \\
\text { Não branco } \\
\text { Branco }\end{array}$ & 0,929 & 0,318 & 8,568 & 1 & 0,003 & $\begin{array}{c}2,533 \\
(1,359-4,719)\end{array}$ \\
\hline $\begin{array}{l}\text { Religiosidade } \\
\text { Não ter religião } \\
\text { Ter religião }\end{array}$ & $-0,673$ & 0,311 & 4,693 & 1 & 0,030 & $\begin{array}{c}0,51 \\
(0,277-0,938)\end{array}$ \\
\hline $\begin{array}{l}\text { Local nascimento } \\
\text { Capital } \\
\text { Interior }\end{array}$ & $-0,829$ & 0,382 & 4,705 & 1 & 0,030 & $\begin{array}{c}0,437 \\
(0,206-0,923)\end{array}$ \\
\hline $\begin{array}{l}\text { Atividade física três ou mais vezes na semana } \\
\text { Sem atividade física } \\
\text { Com atividade física }\end{array}$ & $-1,108$ & 0,307 & 12,997 & 1 & $<0,001$ & $\begin{array}{c}0,33 \\
(0,181-0,603)\end{array}$ \\
\hline Constante & $-0,609$ & 0,521 & 1,368 & 1 & 0,244 & \\
\hline
\end{tabular}

diam ou não estar em estágio de desenvolvimento superior ao da vítima e/ou em uma relação de responsabilidade.

Ademais, notou-se que a variável sofrer violência sexual na infância associou-se à QVI, evidenciando que, mesmo passados muitos anos da vitimização, ela pode continuar impactando negativamente na QV. Esse resultado foi semeIhante ao que a literatura científica aponta. Risman et al. ${ }^{19}$,em um estudo sobre abuso sexual intrafamiliar, apontaram que não é incomum que vítimas apresentem sequelas da violência após muitos anos do ocorrido, e muitas vezes elas apenas compreendem a dimensão do abuso que sofreram quando adultas. Fergusson et al. ${ }^{20}$ apresentaram que violência sexual na infância se associa à depressão, à ansiedade e à ideação/ tentativa de suicídio na vida adulta. Rinfret-Raynor e Cantin ${ }^{21}$ e Thorne-Finch ${ }^{22}$, em estudo com vítimas de violência, apontaram que os sentimentos depressivos, de baixa autoestima e de apatia, gerados pela violência, dificultam a busca de resoluções para o sofrimento, o que explica o fato de as vítimas na infância ainda apresentarem efeitos quando adultos. O estudo de Al-Fayez et al. ${ }^{23}$ com 4.467 estudantes do ensino secundário do Kuwait avaliou a prevalência de abuso sexual e impacto na QV. Os resultados apontaram correlações negativas e significativas entre escores de abuso e domínios de QV (rho $>0,25, p<0,001)$.

Apesar do resultado encontrado neste estudo mostrando associação entre sofrer violência sexual na infância e QVI na vida adulta, não se deve considerar como uma certeza. $\mathrm{O}$ impacto da violência depende de vários fatores, os quais não foram investigados neste estudo: tempo de exposição à agressão, idade da vítima, tipo de abuso, nível de relação com a agressor, suporte social e profissional recebido, entre outros ${ }^{24,25}$.

Em relação às outras variáveis estudadas - sexo, idade, exercer atividade remunerada, apresentar doença crônica, ter tido necessidade de consultas com psiquiatra, ter feito uso de medicação psiquiátrica e apresentar deficiência -, elas não foram associadas significativamente à QV em universitários. Algumas delas estariam associadas a menor QV: ser mulher, segundo Silva e Heleno ${ }^{26}$, trabalhar enquanto cursa a universidade ${ }^{27}$, apresentar doenças crônicas ${ }^{28}$ e apresentar problemas de saúde mental ${ }^{29}$. Porém, nenhum desses estudos realizou análises estatísticas mais robustas como regressão, utilizando em sua maioria teste qui-quadrado e teste t, dificultando comparações com os resultados do presente estudo.

Hipotetiza-se que, para os universitários de uma instituição pública, as questões de gênero, tão comuns na sociedade (machismo, desrespeito à mulher etc.), foram amenizadas pelo maior acesso à educação. $O$ estudo de Silva e Heleno ${ }^{26} \mathrm{com}$ 257 estudantes universitários sobre QV e o bem-estar subjetivo apresentou que maior satisfação com a vida foi encontrada nos estudantes do gênero masculino. No entanto, a presente pesquisa não apresentou diferenças significativas entre os sexos.

Já em relação à idade, o grupo pesquisado era bastante homogêneo, de modo que estar na faixa etária abaixo ou igual a 20 ou acima de 21 não fez diferença estatisticamente significa- 
tiva para a QV, uma vez que a maior parte era de jovens. Quanto ao fato de exercer atividade remunerada não ter influenciado na QV, algumas considerações podem ser realizadas: (a) muitos participantes afirmaram exercer essa atividade mesmo quando eram bolsistas de extensão, pesquisa ou monitoria, a qual envolve somente 8 horas semanais de atividades; (b) não se questionou sobre as condições de trabalho, de modo que os alunos podem ter baixa carga horária ocupacional devido à lei do estágio e estar empolgados pelo primeiro emprego, pelo fato de a maioria ser de jovens universitários. Conforme o Ministério da Saúde ${ }^{30}$, há diversos fatores que devem ser levados em consideração para avaliar os riscos à saúde de uma ocupação, tais como: insalubridade, carga horária total, período, entre outros que neste estudo não foram avaliados.

Segundo Noce et al. ${ }^{31}$, as pessoas portadoras de deficiência física podem apresentar níveis de sedentarismo elevados, influenciando de forma decisiva na percepção de sua QV. No entanto, neste estudo, foram poucos os indivíduos com tipos mais graves de deficiência e houve quem tivesse miopia leve e assinalou a questão. Esse fato revela o quanto as políticas de acessibilidade à educação estão sendo pouco implementadas, ainda que existam leis que instituem igualdade de condições, como a Lei no 13.146, de 6 de julho de 2015.

Em relação às etnias, o grupo dos não brancos, compostos em sua maioria por pardos e negros, apresentou maior associação com QVI. Esses dados são semelhantes aos americanos, visto que a revisão de Pereira et al. ${ }^{32}$ apontou que a maioria dos estudos sugere que negros têm pior QV. Assim, é perceptível que a não igualdade de condições entre brancos e não brancos ainda existe na sociedade brasileira, permeada pelo preconceito, e acarreta a dificuldade de acesso a vários direitos ${ }^{33}$.

Em relação ao nascer no interior apresentar maior associação com QVI, não foram encontrados estudos que façam essa relação. Hipotetiza-se que o menor acesso a serviços de saúde, educação e lazer, quando se vive no interior, explicaria esse resultado. A revisão integrativa de Scorsolini-Comin e Santos ${ }^{34}$ apresenta a importância da satisfação conjugal para a QV, apontando que as pessoas que não estão em relacionamentos apresentam maior associação com QVI.

Já em relação à associação entre ter uma religião e QVI, os resultados deste estudo são discordantes com a literatura. Há estudos que apontam a inter-relação entre o bem-estar espiritual e a saúde gera|35-37. É possível também que este resultado seja singular ao público universitário, pois ele é considerado um dos mais secularizados ${ }^{38}$, sendo importantes estudos futuros sobre o tema.

Outra variável que necessita de mais investigações se refere ao fato de a necessidade de atendimento psicológico ter tido maior associação com QVI. Peres et al. ${ }^{39}$ apontaram que o trabalho terapêutico melhora a QV ${ }^{39}$, mas Padovani et al. ${ }^{40}$ relataram que universitários costumam desvalorizar sintomas, levando a identificação tardia e, muitas vezes, a tratamentos inadequados. Assim, muitas vezes, os que procuram atendimento psicológico podem ter buscado ajuda profissional por conta de sintomas mais graves e neste estudo não se questionou a motivação para os atendimentos psicológicos.

Esperava-se no presente estudo que praticar atividade física ao menos três vezes na semana estaria associado à QVS, o que não foi percebido, contrariando alguns estudos ${ }^{41,42}$. Oliveira et al. ${ }^{43}$ sugerem ser importante não apenas se avaliar se há prática de atividade física, mas também o tipo e a intensidade, se há orientação para essa prática, a finalidade do exercício físico, a existência de lesões devidas a uma prática incorreta, entre outros. É necessário que se realizem outros estudos sobre a relação entre atividade física e QV de universitários, uma vez que eles podem estar realizando uma prática excessiva pela busca do corpo perfeito, devido a pressões sociais, o que indicaria uma percepção de QVI.

Este estudo apresenta algumas limitações. Não há consenso quanto ao ponto de corte do instrumento WHOQOL-bref para delimitar QV inferior e superior, de modo que no presente estudo foram utilizados arbitrariamente os critérios da média e desvio-padrão. O fato de não terem sido analisados o curso, o período e o turno dos estudantes é outra limitação, pois Oliveira ${ }^{44}$ e Catunda e Ruiz ${ }^{27}$ apontaram esses fatores como variáveis importantes na QV dos alunos. Outra limitação é a pequena quantidade de participantes que, após a divisão da amostra pelo escore de QV foram incluídos nos extremos de QVI e QVS, sendo importante em estudos futuros incluir uma amostra maior e mais diversa, também com alunos de universidades particulares.

\section{CONCLUSÕES}

Este estudo teve como objetivo investigar se ser vítima de abuso sexual na infância se relaciona à QV quando adulto, considerando também a influência de outros aspectos, como sexo, prática de atividade física, entre outros. Foi possível notar que ser vítima na infância influenciou na QV atual, sendo muito importante que haja políticas públicas para prevenir, intervir e remediar esse tipo de violência. É também essencial que se amplie a discussão sobre o ASI e a necessidade de formar os profissionais e serviços de saúde para atenção e cuidado integral das vítimas a curto e longo prazo.

Apesar das limitações deste estudo, esta pesquisa é pioneira na análise da QV de estudantes universitários no Ceará e no Brasil. É um dos poucos estudos que empregou análises estatísticas mais complexas e que estudou a relação entre abuso sexual na infância e QV adulta. Ainda assim, é imprescindível o desenvolvimento de outros estudos sobre a temática, por exemplo, com universitários de outras regiões do país, com a população em geral, entre outros. 


\section{CONTRIBUIÇÕES INDIVIDUAIS}

Karla Julianne Negreiros de Matos - Concepção do projeto, coleta, digitação e análise dos dados, interpretação dos resultados e redação do manuscrito.

Francisco José Maia Pinto - Análise dos dados, revisão crítica relevante do conteúdo intelectual e correção do manuscrito.

Ana Carina Stelko-Pereira - Concepção do projeto, análise dos dados e interpretação dos resultados, revisão crítica relevante do conteúdo intelectual e redação do manuscrito.

\section{REFERÊNCIAS}

1. World Health Organization. Guidelines for medico-legal care for victims of sexual violence. Geneva: WHO; 2003.

2. Ministério do Planejamento, Desenvolvimento e Gestão. Instituto Brasileiro de Geografia e Estatística (IBGE). Diretoria de Pesquisas. Coordenação de População e Indicadores Sociais. Pesquisa nacional de saúde do escolar: 2015/IBGE, Coordenação de População e Indicadores Sociais. Rio de Janeiro: IBGE; 2016. 132p.

3. Guedes A, Bott S, Garcia-Moreno C, Colombini M. Bridging the gaps: a global review of intersections of violence against women and violence against children. Glob Health Action. 2016;9:31516.

4. Martins CBG, Jorge MHPM. Abuso sexual na infância e adolescência: perfil das vítimas e agressores em município do sul do Brasil. Texto Contexto Enferm. 2010;19(2):246-55.

5. Machado HB, Lueneberg CF, Régis El, Nunes MPP. Abuso sexual: diagnóstico de casos notificados no município de Itajá/SC, no período de 1999 a 2003, como instrumento para a intervenção com famílias que vivenciam situações de violência. Texto Contexto Enferm. 2005;14(spe):54-63.

6. Ribeiro WS, Andreoli SB, Ferri $(P$, Prince $M$, Mari JJ. Exposição à violência e problemas de saúde mental em países em desenvolvimento: uma revisão da literatura. Rev Bras Psiquiatr. 2009;31(Sup| II):S49-57.

7. Speetjens $P$, Thielen F, Ten Have M, de Graaf R, Smit F. [Child maltreatment: long-term economic consequences and implications]. Tijdschr Psychiatr. 2016;58(10):706-11.

8. Leeb RT, Lewis T, Zolotor AJ. A review of physical and mental health consequences of child abuse and neglect and implications for practice. Am J Lifestyle Med. 2011;5(5):454-68.

9. Weber S, Jud A, Landolt MA. Quality of life in maltreated children and adult survivors of child maltreatment: a systematic review. Qual Life Res. 2016;25(2):237-55.

10. Oviedo-Joekes E, Marchand K, Guh D, Marsh DC, Brissette S, Krausz M, et al. History of reported sexual or physical abuse among long-term heroin users and their response to substitution treatment. Addict Behav. 2011;36(1-2):55-60.

11. The World Health Organization Quality of Life assessment (WHOQOL): position paper from the World Health Organization. Soc Sci Med. 1995;41(10):1403-9.

12. Cirino S, et al. Anais Simpósio de Pesquisa e Intercâmbio Científico (14:2012, Belo Horizonte, MG) Anais do XIV Simpósio de Pesquisa e Intercâmbio Científico: Produção e Divulgação Científica: os desafios da interdisciplinaridade. Belo Horizonte: ANPEPP, 2012. p. 23-6.

13. Fleck MPA, Louzada S, Xavier M, Chachamovich E, Vieira G, Santos L, et al. Aplicação da versão em português do instrumento abreviado de avaliação da qualidade de vida "WH000L-bref". Rev Saúde Pública. 2000;34(2):178-83.

14. Bowden A, Fox-Rushby JA. A systematic and critical review of the process of translation and adaptation of generic health-related quality of life measures in Africa, Asia, Eastern Europe, the Middle East, South America. Soc Sci Med. 2003;57(7):1289-306.

15. Dantas RAS, Sawada NO, Malerbo MB. Pesquisas sobre qualidade de vida: revisão da produção científica das universidades públicas do Estado de São Paulo. Rev Latino-Am Enfermagem. 2003;11(4):532-8

16. Fleck MPA, Borges ZN, Bolognesi G, Rocha NS. Desenvolvimento do WH0Q0L, módulo espiritualidade, religiosidade e crenças pessoais. Rev Saúde Pública. 2003;37(4):446-55.

17. Matos KJN, Stelko-Pereira AC. Análise aparente de tecnologia educativa para universitários sobre abuso sexual infantil. Psico-USF [online]. 2015;20(2):349-52.

18. Instituto Brasileiro de Geografia e Estatística (IBGE). Censo 2010. Disponível em: <http:// www.censo2010.bge.gov.br/sinopse/index.php?dados=8>. Acesso em: 10 maio 2016.
19. Risman ARL, Figueira G, Mendeiros V, Teixeira LA. Abuso sexual intrafamiliar: una mirada multifacetica al incesto. Psicol Am Lat. 2014:88-100.

20. Fergusson DM, Boden JM, Horwood LJ. Exposure to childhood sexual and physical abuse and adjustment in early adulthood. Child Abuse Negl. 2008;32(6):607-19.

21. Rinfret-Raynor M, Cantin S. Violence Conjugale rapportée par des femmes s'adressant aux services sociaux. In: Violence conjugale: recherches sur la violence faite aux femmes en milieu conjugal. Boucherville, Québec: G. Morin; 1994. p. 3-22.

22. Thorne-Finch R. Ending the silence: the origins and treatment of male violence against women. Buffalo: University of Toronto Press; 1992.

23. Al-Fayez GA, Ohaeri JU, Gado OM. Prevalence of physical, psychological, and sexual abuse among a nationwide sample of Arab high school students: association with family characteristics, anxiety, depression, self-esteem, and quality of life. Soc Psychiatry Psychiatr Epidemiol. 2012;47(1):53-66.

24. Baía PAD, Veloso MMX, Magalhães CMC, Dell'Aglio DD. Caracterização da revelação do abuso sexual de crianças e adolescentes: negaçãa, retratação e fatores associados. Temas Psicol. 2013;21(1):193-202.

25. Von Hohendorff J, Costa LS, Habigzang LF, Koller SH. Análise documental de casos de violência sexual contra meninos notificados em Porto Alegre. Paidéia (Ribeirão Preto). 2014;24(58):13-8.

26. Silva EC, Heleno MGV. Qualidade de vida e bem-estar subjetivo de estudantes universitários. ver Psicol Saúde. 2012:4(1):69-76.

27. Catunda MAP, Ruiz VM. Qualidade de vida de universitários. Pensamento Plural: Revista Científica do UNIFAE. 2008;2(1):22-31.

28. Santos AKGV, Reis CC, Chaud DMA, Morimoto JM. Qualidade de vida e alimentação de estudantes universitários que moram na região central de São Paulo sem a presença dos pais ou responsáveis. Rev Simbio-Logias. 2014;7(10):76-99.

29. Cerchiari EAN. Saúde mental e qualidade de vida em estudantes universitários [tese]. Campinas, SP: Universidade Estadual de Campinas; 2004

30. Brasil. Ministério da Saúde. Secretaria de Vigilância em Saúde. Secretaria de Atenção à Saúde. Política Nacional de Promoção da Saúde: PNPS: revisão da Portaria MS/GM no 687, de 30 de março de 2006. Braślia: Ministério da Saúde; 2015.

31. Noce F, Simim MAM, Mello MT. A percepção de qualidade de vida de pessoas portadoras de deficiência física pode ser influenciada pela prática de atividade física?. Rev Bras Med Esporte. 2009;15(3):174-8

32. Pereira CCA, Palta M, Mullahy J. Health domains and race in generic preference-based health-related quality of life instruments in the United States literature. Rev Bras Estud Popul. 2010;27(2):425-37.

33. Guimarães ASA. Acesso de negros às universidades públicas. Cad Pesq. 2003;118:247-68

34. Scorsolini-Comin F, Santos MA. Satisfação conjugal: revisão integrativa da literatura científica nacional. Psic Teor Pesq. 2010;26(3):525-32.

35. Costa CC, Bastiani M, Geyer JG, Calvetti PÜ, Muller MC, Moraes MLA. Qualidade de vida e bem-estar espiritual em universitários de psicologia. Psicol Estud. 2008;13(2):249-55.

36. Contrada RJ, Goyal TM, Cather C, Rafalson L, Idler EL, Krause TJ. Psychosocial factors in outcomes of heart surgery: the impact of religious involvement and depressive symptoms. Health Psychol. 2004;23(3):227-38.

37. Freedland KE. Religious beliefs shorten hospital stays? Psychology works in mysterious ways: comment on Contrada et al. (2004). Health Psychol. 2004;23(3):239-42.

38. Ribeiro JC. Georg Simmel, pensador da religiosidade moderna. Rev Estud Religião. 2006;(2):109-26

39. Peres JFP, Simão MJP, Nasello AG. Espiritualidade, religiosidade e psicoterapia. Rev Psiquiatr Clín. 2007;34(Supl 1):136-45.

40. Padovani RC, Neufeld CB, Maltoni J, Barbosa LNF, Souza WF, Cavalcanti HAF, et al. Vulnerabilidade e bem-estar psicológicos do estudante universitário. Rev Bras Ter Cogn. 2014;10(1):2-10

41. CieslakF, Cavazza JF, Lazarotto L, Titski ACK, Stefanello JMF, Leite N. Análise da qualidade de vida e do nível de atividade física em universitários. Rev Educ Fis. UEM. 2012;23(2):251-60.

42. Mendes Netto RS, Silva CS, Costa D, Raposo OFF. Nível de atividade física e qualidade de vida de estudantes universitários da área de saúde. Rev Bras Ciênc Saúde. 2012:10(34):47-54.

43. Oliveira CS, Gordia AP, Quadros TMB, Campos W. Atividade física de universitários brasileiros: uma revisão da literatura. Rev Atenção Saúde. 2014;12(42):71-7.

44. Oliveira JAC. Qualidade de vida e desempenho acadêmico de graduandos [tese]. Campinas: Universidade Estadual de Campinas (Unicamp); 2006 